\title{
Culture scientifique et encourage- ment de la relève en médecine
}

\author{
Hermann Amstad, MPH
}

Dr med., Secrétaire général de I'ASSM

Voici quelques années que la qualité de la recherche médicale soulève de nombreuses critiques; en même temps, la pénurie de jeunes chercheurs dans le domaine de la médecine académique représente un problème croissant. Dans une nouvelle feuille de route*, l'Académie Suisse des Sciences Médicales (ASSM) propose une série de mesures destinées à améliorer aussi bien la culture scientifique que la situation de la relève scientifique.

La pénurie de médecins fait actuellement l'objet de nombreuses discussions et concerne tout particulièrement la médecine de premier recours. Le manque de relève dans la recherche en médecine académique est moins débattu, bien qu'il représente un problème croissant depuis des années. Dans sa feuille de route «La médecine comme science», l'ASSM avait proposé dès 2009 des mesures concrètes pour augmenter l'attractivité des carrières orientées vers la recherche en sciences biomédicales. Certes, la promotion de la relève a été améliorée dans certains hôpitaux universitaires et universités (par ex. "protected research time» pendant la formation postgraduée), mais, depuis quelques années, la qualité de la recherche biomédicale soulève de plus en plus de critiques. Des voix s'élèvent haut et fort pour exiger des résultats d'études médicales plus parlants et réduire le nombre d'études inutiles: «increasing value - reducing waste». Pour ce faire, il importe d'élaborer un programme de "scientific change» qui, grâce à une meilleure prise en compte des connaissances existantes et à la planification et à la réalisation minutieuse des études scientifiques, contribue à éviter le gaspillage des ressources financières, structurelles et humaines en pratiquant des examens peu parlants.

On peut supposer qu'il existe une relation directe entre une culture scientifique intègre et digne de confiance et la motivation de la relève pour une carrière dans la recherche biomédicale. C'est pourquoi l'ASSM a remis ce sujet à l'ordre du jour. Elle a instauré un groupe de travail, spécialement chargé d'étudier la situation en Suisse. Selon ce groupe de travail, pour promouvoir la relève scientifique, des mesures s'imposent dans l'en- semble du domaine de la recherche biomédicale, c'està-dire de la recherche clinique fondamentale, de la recherche translationnelle et de la recherche orientée principalement vers le patient. Il propose des mesures urgentes pour encourager les médecins chercheurs, "the newest endangered species", en se référant à d'anciennes publications, notamment à la feuille de route «La médecine comme science», au rapport du groupe thématique "Relève pour la recherche clinique en Suisse» et aux "Recommandations pour des carrières académiques intégrant la dimension d'égalité».

\section{Des mesures s'imposent dans l'ensemble} du domaine de la recherche biomédicale.

Avec cette nouvelle feuille de route, on dispose de recommandations actualisées pour un encouragement efficace de la relève et des carrières des médecins chercheurs. Toutefois, au courant des discussions, il s'est avéré clairement que le problème de fond de la culture scientifique déficiente concerne autant d'autres secteurs académiques que les sciences biomédicales. Dès lors, les Académies suisses des sciences ont repris le thème de la culture scientifique pour le traiter plus largement; l'ASSM poursuit, elle aussi, son engagement dans ce sens.

\section{Mesures dans le domaine de la culture scientifique}

1. Les instituts de recherche doivent instaurer les règles de "good research practice» (GRP) et de "good clinical practice» (GCP), veiller à leur applica- 
tion et créer des organismes et des antennes chargés de déceler d'éventuelles infractions.

2. Seuls les résultats de recherche ayant été validés au sein du groupe de recherche peuvent être communiqués vers l'extérieur; ce faisant, un soin particulier doit être apporté aux détails méthodologiques. L’accès aux données originales doit être garanti et même les résultats négatifs doivent être accessibles au public.

3. Les hôpitaux universitaires doivent communiquer le plus tôt possible à leurs jeunes chercheurs que le financement des activités de recherche est indépendant des prestations, et les soutenir lors de l'acquisition de fonds pour la recherche.

4. Dans l'évaluation de la personnalité individuelle d'un chercheur, il importe de ne pas uniquement tenir compte des publications, mais également d'autres facteurs, tels que les activités d'enseignement, l'acquisition de fonds étrangers, le potentiel d'innovation, le plan de carrière, éventuellement les compétences cliniques, l'observance des règles de l'intégrité scientifique, la capacité de travailler en équipe et les obligations familiales.

5. Une promotion de la relève scientifique durable et soucieuse de l'égalité des genres doit faire partie intégrante d'une culture scientifique responsable; elle définit en grande partie l'attractivité d'une carrière scientifique.

\section{Mesures dans le domaine de l'encourage- ment de la relève}

1. Les programmes MD-PhD et Dr sc. med. offrent aux médecins chercheurs une formation idéale et doivent bénéficier d'un soutien prioritaire par des fonds nationaux et locaux.

2. Les hôpitaux universitaires et les hôpitaux cantonaux avec une activité de recherche devraient aménager les horaires des médecins assistants en faveur d'une activité de recherche pendant leur formation postgraduée ("protected research time»).

3. Les facultés de médecine sont invitées à coordonner le modèle de promotion de la relève du Fonds National Suisse (SNF) - qui est cohérent et logiquement structuré - avec la formation postgraduée clinique et à l'ancrer dans les hôpitaux universitaires.

4. En plus des quelques rares postes de médecins-chefs, les hôpitaux universitaires doivent offrir aux médecins chercheurs des options de carrière attractives et favorables à la vie de famille. Concrètement, le modèle américain "attending physician system» pourrait être adopté et/ou au minimum le "dual leadership» pourrait être introduit dans les cliniques spécialisées: la séparation de la gestion et des responsabilités entre la clinique et la recherche avec des échanges réciproques et des possibilités de rotation.

5. Dans les procédures de nomination, les performances dans la promotion de la relève devraient faire partie des principaux critères d'évaluation. Le potentiel individuel pour la clinique, la recherche et l'enseignement est révélé par le nombre et la qualité des jeunes chercheurs formés par un candidat à un poste de professeur.

\section{Adaptation des structures d'organisation}

La médecine académique porte l'empreinte des personnes et de leur culture d'une part, et celle des structures dans laquelle elle est exercée d'autre part. Les conditions et les mesures destinées à encourager la culture scientifique et la relève, telles qu'elles sont décrites ci-dessus, doivent également se refléter dans les structures d'organisation des institutions.

Dans les hôpitaux universitaires, les contraintes économiques risquent de marginaliser la culture scientifique. Si les hôpitaux universitaires veulent être à la hauteur de leur rôle de leader dans le domaine de la médecine tertiaire, le mandat de recherche et d'enseignement universitaire doit également se refléter dans les structures de gestion.

Les hôpitaux universitaires devraient être dirigés par des personnalités académiques ayant une large expérience en clinique, recherche et management. L'organisation tient compte d'une répartition adéquate des tâches entre les prestations, la recherche, l'enseigne-

\section{Les prestations médicales et le travail scienti-}

fique doivent être reconnus comme équivalents.

ment et le management. Tout particulièrement dans les domaines d'activités prioritaires, la recherche et les prestations devraient être dirigées par des personnes différentes qui coopèrent étroitement selon le principe $\mathrm{du}$ "dual leadership».

Les prestations médicales et le travail scientifique doivent être reconnus comme équivalents et interagir aussi étroitement que possible, au sens de la recherche translationnelle. A l'ère de «Big Data - Big Health» il est indispensable de disposer de structures de recherche dépassant le cadre des services et des cliniques, qui permettent un accès aux systèmes d'informations cliniques, aux banques de données et aux biobanques. La recherche ainsi que les postes de chercheurs ne pouvant pas bénéficier de financements croisés, les 
hôpitaux universitaires sont tributaires de soutiens spéciaux de la part des universités. Les facultés de médecine doivent veiller à ce que les hôpitaux universitaires disposent de structures de recherche adéquates qui encouragent une haute culture scientifique. Ceci englobe des critères tels que le respect des principes GRP et GCP, l'intégrité dans la science, une gestion efficace des erreurs, le travail d'équipe, l'ouverture et la confiance, une relève scientifique respectant l'équilibre entre femmes et hommes, la reconnaissance équivalente de l'activité clinique et de la recherche pendant la formation postgraduée.

Les universités soutiennent les hôpitaux universitaires dans l'établissement de postes de formation postgraduée incluant le "protected research time» comme partie intégrante du profil. Elles établissent, en

\section{Les hôpitaux universitaires sont tributaires de soutiens spéciaux de la part des universités.}

collaboration avec le Fonds National Suisse, des plans de carrière réalistes permettant aux médecins chercheurs de trouver leur équilibre entre la clinique, la recherche et la vie de famille et, à long terme, de rester éligible à des postes de cadres en médecine académique. Les plans de carrière dans les hôpitaux universitaires devraient être conçus de manière plus flexible et multidimensionnelle comme dans les systèmes anglo-saxons/américains, par exemple par l'introduction du système "attending physician" ou par des chaires professorales précliniques avec une responsabilité clinique partielle dans des domaines spécifiques.

Afin d'encourager l'établissement de structures d'organisation adéquates, les institutions (cliniques, services, groupes de recherche) avec une excellente culture de promotion de la relève et de la science devraient être distinguées respectivement accréditées. Pour ce faire, le FNS, l'ASSM, l'association de la médecine universitaire ou une autre organisation académique disposeraient des moyens nécessaires.

\section{Perspective}

La feuille de route s'inscrit dans l'engagement de longue date de l'ASSM en faveur d'une meilleure culture de la recherche et de l'encouragement de la relève en médecine académique. Elle constate le lien entre les déficits de la culture de recherche biomédicale largement débattus au niveau international et la diminution manifeste de la motivation des jeunes médecins pour une carrière scientifique. A cela s'ajoutent les enjeux multiples et cumulatifs de l'activité clinique, de la recherche et de la vie de famille, qui ne permettent que difficilement aux médecins chercheurs de trouver un équilibre entre vie professionnelle et vie privée.

Il va de soi que la pénurie actuelle de la relève dans tous les secteurs de la recherche biomédicale (fondamentale, translationnelle et clinique) s'amplifiera si l'on ne réussit pas à améliorer l'attractivité d'une carrière de médecin chercheur à l'aide de mesures efficaces. Ce constat a été confirmé tant au niveau national qu'international. Ainsi, dans le cadre de Horizon 2020, l'UE attribue une importance majeure au thème de la promotion de la relève intégrant la dimension d'égalité des sexes. En Suisse, la promotion de la relève dans la recherche biomédicale, et notamment dans la recherche clinique, a été inscrite dans l'agenda politique, suite au rapport de la plateforme thématique «Avenir de la formation médicale» co-rédigé par l'ASSM et aux pressions de l'industrie pharmaceutique.

La réalisation de ces mesures multidimensionnelles met au défi toutes les institutions du système de formation et de santé suisse. L'ASSM est prête à affronter ces défis et à contribuer activement à l'amélioration de la culture scientifique, de l'encouragement de la relève et des structures d'organisation dans le domaine de la médecine académique. Elle considère les réformes proposées comme incontournables et importantes; elle instaurera un groupe de travail chargé du suivi de la mise en œuvre des mesures proposées et du soutien des institutions concernées. 